\title{
HIGH-MOUNTAIN HYBRID INSTALLATION FOR MULTICOMPONENT DETECTION OF AIR-SHOWERS INDUCED BY ULTRA-HIGH ENERGY COSMIC RAYS
} \author{
Zhumabayev $^{a}$, P Bezyazeekov ${ }^{g}$, Oleg Fedorov ${ }^{g}$. \\ ${ }^{a}$ al-Farabi Kazakh National University, \\ 050040, al-Farabi, 71, Almaty, Kazakhstan \\ ${ }^{b}$ P.N. Lebedev Physical Institute of the Russian Academy of Sciences, \\ 119991, Leninsky avenue, 53, Moscow, Russia \\ ${ }^{c}$ Clayton State University, 2000 Clayton State Blvd., Morrow GA 30260, USA \\ ${ }^{d}$ Joint Institute for Nuclear Research, \\ 141980, str. Joliot-Curie, 6, Dubna, Russia \\ ${ }^{e}$ Institute of Nuclear Physics \\ 1 Ibragimova St, Almaty, 050032, Republic of Kazakhstan \\ ${ }^{f} D E S Y$ \\ 15738 Zeuthen, Germany \\ ${ }^{g}$ API ISU, \\ Irkutsk, 6640 b Russia \\ *Degtyareva street 11, Almaty, Kazakhstan \\ Al-Farabi Kazakh National University, Physics-tehnical faculty \\ Al-Farabi av. 71/23, Almaty, Kazakstan \\ *E-mail: saken199303@mail.ru
}

Saken Shinbulatov ${ }^{a, b},{ }^{*}$ Nurzhan Saduyev ${ }^{a, b}$, Dmitriy Beznosko ${ }^{c}$, Orazaly Kalikulov ${ }^{a}$, Valeri $^{2}$ Zhukov $^{b}$, Yerzhan Mukhamejanov a,d,e, Dmitriy Kostunin ${ }^{f}$, Beibit Karibayeva, Timur Namazbayeva $^{a}$ Aliya Baktoraz, Nurzhan Yerezhep ${ }^{a, b}$, Shynbolat Utey ${ }^{a}$, Askhat

In this work, we discuss deployment of a new setup of high-frequency antennas for detection of air showers. It is considered to employ two types of high-frequency antennas for the new setup, the first type of antenna is the SALLA type, which is used in the Tunka-Rex experiment, and the second type is a narrow-radiational high-frequency Uda-Yagi antenna. The comparison of radiation patterns, frequency range and noise level in the mountains and at the university altitude levels is presented.

37th International Cosmic Ray Conference (ICRC 2021)

July 12th-23rd, 2021

Online-Berlin, Germany 


\section{Introduction}

It is planned to create a new experimental setup consisting of high-frequency antennas at the $3340 \mathrm{~m}$ above sea level at the Tien Shan High-altitude Scientific Station (TSHSS) near Almaty city (Kazakhstan).

In perspective, all experimental setups at TSHSS will be combined into a single complex. The modernized complex installation will detect the fluxes of charged particles and radio emission from Extended Air Showers (EAS). Space-time distributions of charged particles fluxes of EAS will be studied using the data obtained by the complex installation.

In this work, it is proposed to use two types of high-frequency antennas, such as SALLA, and a narrow-beam Yagi-Uda antenna prototype for operation in a tandem [1].

The electronic part of the narrow-beam antennas was designed and assembled at the alFarabi Kazakh National University and was combined with the SALLA type antennas.

\section{Setting up an experimental and comparative analysis}

Radio pulses from EAS generated by the showers caused by ultra-high energy astroparticles (e.g. cosmic rays, gamma quanta and neutrinos) are formed due to the curvature of electrons trajectories and positrons in the shower front due to the Earth's magnetic field. These pulses are broadband radiation in the $\mathrm{MHz}$ range and are narrow bursts of tens of nanoseconds duration. Antenna arrays equipped with electronics with high sampling rate and a large data buffer are used to record these pulses [2]. Typically, such experiments use flash ADC (analog-to-digital converters) with a sampling rate of at least $200 \mathrm{MHz}$ and a frame size of several thousand samples. An active antenna and a filter amplifier are usually connected to these electronics.

As part of this project, high frequency antennas with a radiation pattern optimized for inclined EAS, were developed. The antennas will be connected to a cascade of amplifiers developed at KIT for Tunka-Rex antennas and provided for TSHSS. The measuring circuit will be connected to a commercially available CAEN ADC DT5725 [3].

Yagi-Uda antenna was created to detect radio emission from EAS in the frequency range of 55-65 MHz. First, a computer model of the antenna was designed and an experimental model (a real prototype) was created based on the simulations.

Figure 1 shows the antenna model created in the HFSS Ansoft environment [4] by the finite element method and shows a prototype antenna. The active vibrator half-wave length $(0.5 \lambda)$ is $2.38 \mathrm{~m}$, the reflector length, slightly larger than $0.5 \lambda$, is $2.45 \mathrm{~m}$, the directors are $2.26 \mathrm{~m}$ and 2.24 $\mathrm{m}$, respectively. All active and passive vibrators are made of aluminum pipe with an outer diameter of $10 \mathrm{~mm}$. The length of the wooden boom (traverse) is $2.5 \mathrm{~m}$. According to the simulation results, frequency parameters and radiational characteristics were obtained. Figure 2 shows the frequency reflection coefficients of the antenna in the band of 30-80 MHz. At the level of "- $10 \mathrm{~dB}$ ", the operating frequencies of the antenna are in the band of $58-62 \mathrm{MHz}$ and the bandwidth is $4 \mathrm{MHz}$. Figure 3 shows the radiation patterns at the central frequency of $60 \mathrm{MHz}$, built by the gain factor. The maximum radiation wave is $9.38 \mathrm{~dB}$. Two-dimensional radiation pattern with the widths of the main lobes in the vertical (810) and horizontal (570) planes are also given. 


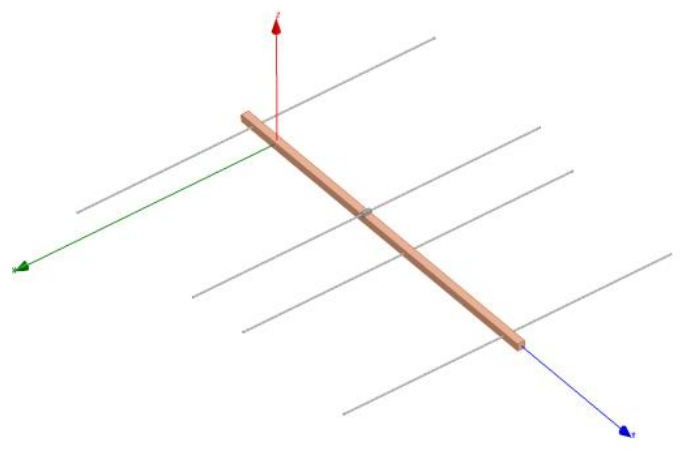

Figure 1. Antenna model in HFSS Ansoft (Yagi-Uda antenna prototype)

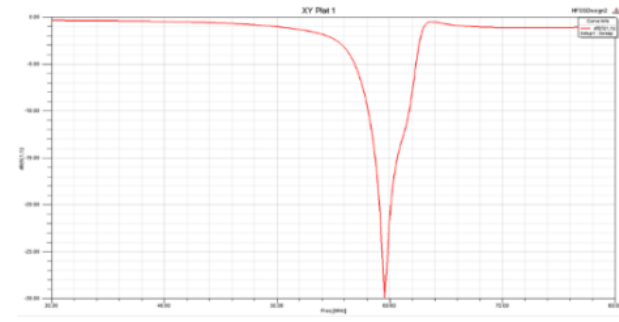

Figure 2. $S_{11}$ parameter (reflection coefficient) in the band of 30-80 MHz (Yagi-Uda antenna prototype)

The geometric and physical parameters of the computer model and its physical prototype fully correspond. An experiment was conducted to determine the antennas characteristics. For this purpose, a high-frequency signal generator G4-158 and Agilent N 9340B [5] spectrum analyzer were used, and measuring devices were connected to the antennas via coaxial cables (50 ohms) with a length of $5 \mathrm{~m}$ with "N male to SMA male" connectors. To study the bandwidth, the signal generator was radiated in the range of 40-65 MHz with a step of $1 \mathrm{MHz}$. The distance between identical antenna samples was $20 \mathrm{~m}$. As a result, the dependence of the received power on the frequency was constructed, which showed that the antenna can receive useful signals in the frequency range from $45 \mathrm{MHz}$ to $63 \mathrm{MHz}$ (Fig. 3d). The radiation pattern was also built in a horizontal plane. The measurement results showed that the antenna is narrowly directed and, in addition to the main lobe, has a relatively small rear lobe (Fig. 3d). The simulation results are in good agreement with the results of field experiments.

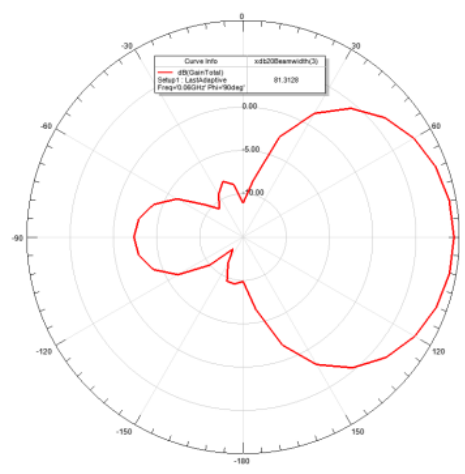

a)

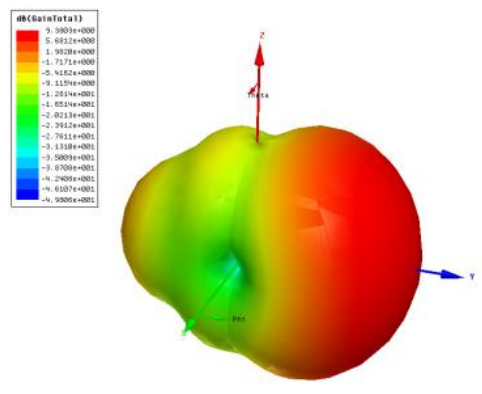

c)

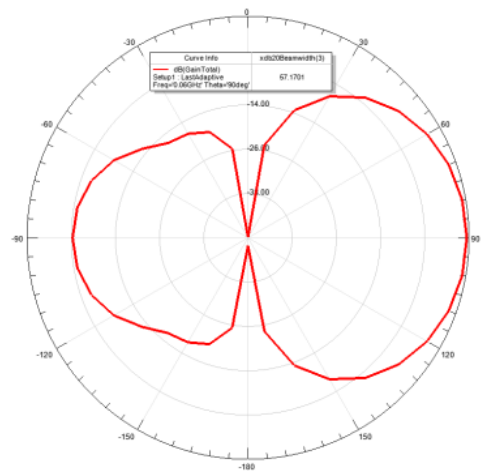

b)

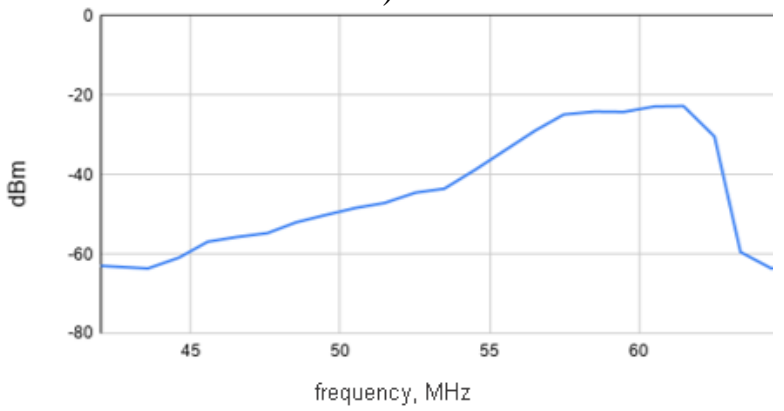

d)

Figure 3. Radiation patterns based on the gain and width of the main lobes in two planes of the Yagi-Uda antennas type $(3 a, 3 b, 3 c)$ and useful signals in the frequency range from $45 \mathrm{MHz}$ to $63 \mathrm{MHz}(3 d)$ 


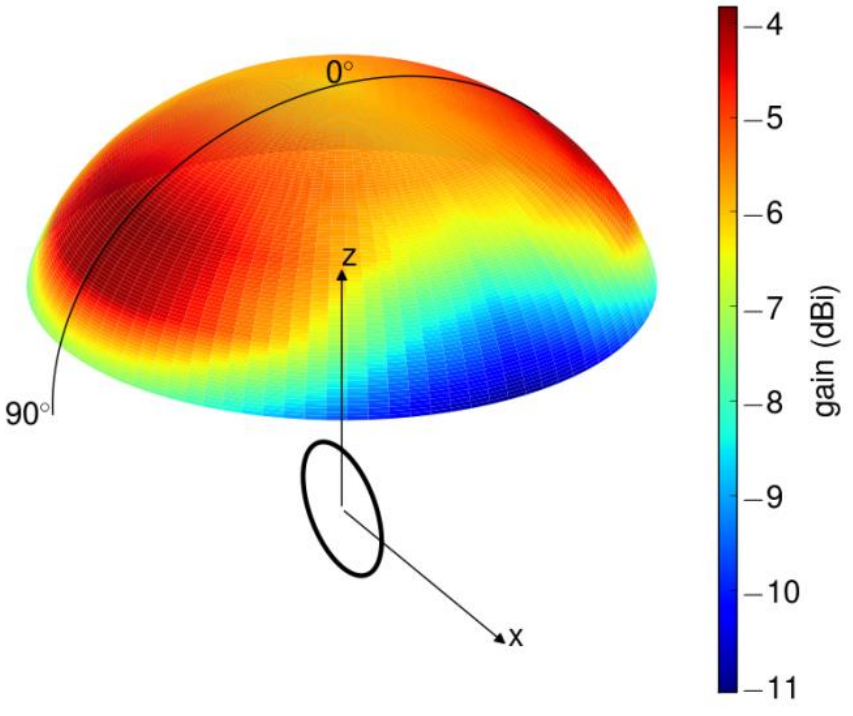

a) radiation pattern

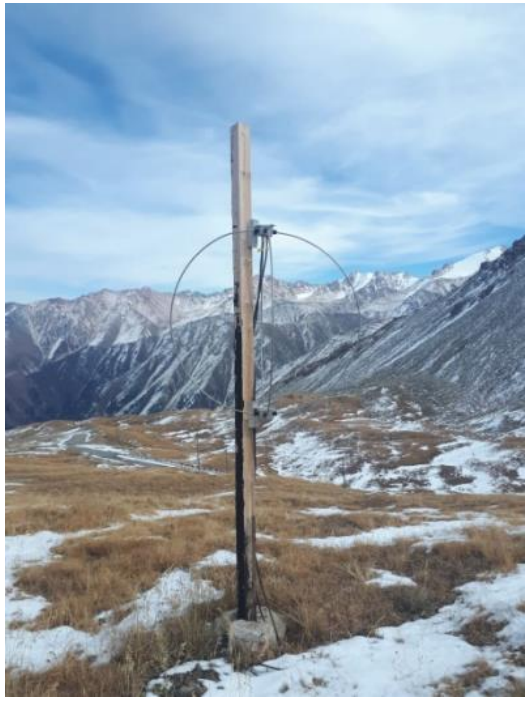

b) SALLA type antenna

Figure 4. The SALLA antenna, which is installed in TSHSS, and the radiation pattern of antennas SALLA type.

For further development of antennas Yagi-Uda type and expansion of the complex installation, calibration will be carried out using SALLA antennas type, which is shown in Figure 4a that shows the radiation pattern of the SALLA type antenna and Figure 4b shows the TSHSS antenna station with its two perpendicularly aligned SALLA antennas [6].

As indicated in Figures 3 and 4, we can notice a comparative difference between antennas SALLA type and antennas Yagi-Uda type.

In the measurements described above, bandpass filters and low-noise amplifiers (LNA) were not used. LNA is usually used to amplify very weak signals. We have studied the amplitude characteristics of a receiving antenna system with LNA and filter. To obtain this, the main active vibrator of the antenna was directly connected to LNA, as well as the antenna cable was connected to the spectrum analyzer through a bandpass filter. The signal with a frequency of $60 \mathrm{MHz}$ was received in three modes: antenna, antenna+LNA, antenna+LNA+filter.

The measurement results are shown in Figure 5 in a spectral analyzer for two conditions: at TSHSS and the University altitude ( $880 \mathrm{~m}$ a.s.1.).

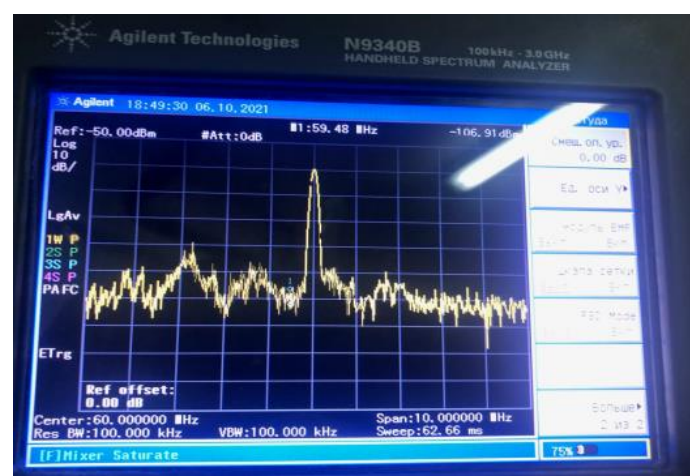

a)

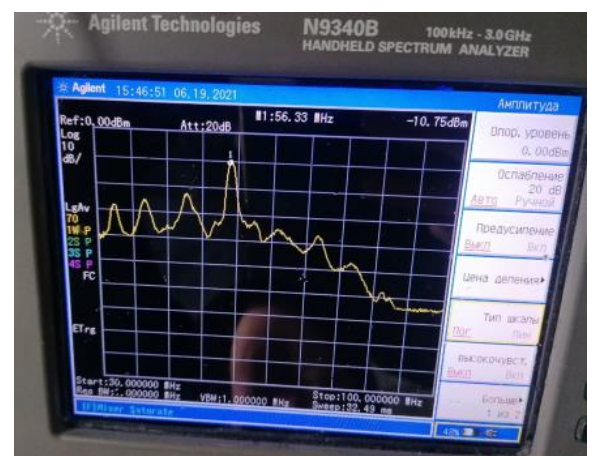

b) 
Figure 5. Spectral analysis $a$ - the University altitude; $b$ - at TSHSS

\section{Summary}

Preliminary results on the prototype Udo-Yagi antenna such as radiation pattern, frequency range and noise level data provide a good prospect for working in tandem with SALLA (using a unified amplifier and bandpass filter)

\section{Acknowledgements}

This work was supported by the grant from the Committee of Science of the Ministry of Education and Science of the Republic of Kazakhstan AP09058049 "Study of extensive air showers with delayed fronts".

\section{References}

[1] T. Marshalkina et al. (Tunka-Rex) "First analysis of inclined air showers detected by Tunka-Rex," EPJ Web Conf. 216, 02012 (2019) doi:10.1051/epjconf/201921602012 [arXiv:1812.03724 [astroph.IM]]

[2] D. Heck, J. Knapp, J. N. Capdevielle, G. Schatz and T. Thouw, “CORSIKA: A Monte Carlo code to simulate extensive air showers," FZKA-6019.

[3] CAEN S.p.A. Via della Vetraia, 11, 55049 Viareggio Lucca, Italy. http://caen.it.

[4] ANSYS HFSS software. http://www.ansoft.com/products/hf/hfss/

[5] N9340B Handheld RF Spectrum Analyzer (HSA), 1400 Fountaingrove Parkway Santa Rosa, CA 95403-1738 https://www.keysight.com/zz/en/contact.html

[6] R. Hiller Radio measurements for determining the energy scale of cosmic rays // Doktors der Naturwissenschaften, - Karlsruher Instituts fur Technologie - 2016 